\title{
AN APPLICATION OF THE DEGENERATE BELTRAMI EQUATION: QUADRATIC POLYNOMIALS WITH A SIEGEL DISK
}

\author{
Liang Shen \\ Beijing Institute of Technology, School of Mathematics and Statistics \\ Beijing 100081, P. R. China; shenl@bit.edu.cn
}

\begin{abstract}
Let $v(t)>0$ be a concave function such that $\int_{1}^{+\infty} \frac{1}{t v(t)} d t=+\infty$. If the continued fraction expansion of an irrational number $0<\theta<1$ has the coefficient $a_{k}$ which satisfies

$$
\log ^{2} a_{k} \leq k v(k), \quad k=1,2, \cdots,
$$

the Julia set of $e^{2 \pi i \theta} z+z^{2}$ is locally connected and has Lebesgue measure zero. It extends the results of Petersen and Zakeri [10].
\end{abstract}

\section{Introduction}

Let $0<\theta<1$ be an irrational number with the continued fraction expansion $\theta=\left[0 ; a_{1}, a_{2}, \cdots\right]$. In [10], Petersen and Zakeri proved that if $\log ^{2} a_{k}=\mathcal{O}(k)$, the Julia set of $P_{\theta}(z)=e^{2 \pi i \theta} z+z^{2}$ is locally connected and has Lebesgue measure zero. In the paper, we extend their results by the following theorem.

Theorem 1.1. Let $v(t)>0$ be a concave function such that $\int_{1}^{+\infty} \frac{1}{t v(t)} d t=+\infty$. If the continued fraction coefficient $a_{k}$ satisfies that

$$
\log ^{2} a_{k} \leq k v(k), \quad k=1,2, \cdots,
$$

the Julia set of $P_{\theta}(z)$ is locally connected and has Lebesgue measure zero.

For example the functions $\log t, \log t \log \log t, \cdots$ satisfy the conditions of the Theorem 1.1.

Corollary 1.2. Let $n$ be a fixed integer. If the continued fraction coefficient $a_{k}$ satisfies that

$$
\log ^{2} a_{k} \leq k \cdot \log k \cdot \log \log k \cdots \underbrace{\log \log \cdots \log k}_{n},
$$

the Julia set of $P_{\theta}(z)$ is locally connected and has Lebesgue measure zero.

The proof of Theorem 1.1 bases on the existence of solutions of the degenerate Beltrami equation developed by Iwaniec and Martin [3, 6, 7]. We first give a reformulation of their theorem, then prove the homeomorphic solution satisfies the Lusin's condition $\mathcal{N}$. Finally we can prove the theorem by a classical argument. The area estimation in [10] given by Petersen and Zakeri is important for us.

https://doi.org/10.5186/aasfm.2018.4311

2010 Mathematics Subject Classification: Primary 30C62, 30C65, 35J70.

Key words: Beltrami equation, mapping of finite distortion, Siegel disk.

The author would like to thank the referees for careful reading of the manuscript and many valuable suggestions. 


\section{Definitions and known results}

In this section we introduce some definitions and known results.

Let $\mu(z)$ be a measurable function compactly supported in the plane $\mathbf{C}$ and $|\mu(z)|<1$ almost everywhere. If the distortion function $K(z)=\frac{1+|\mu(z)|}{1-|\mu(z)|}$ has an upper bound, the existence of the solution of the the Beltrami equation $f_{\bar{z}}=\mu(z) f_{z}$ is well-known by the measurable Riemann mapping theorem, see $[1,8]$. When $K(z)$ is unbounded, the following well-known theorem was given by David [4], see also [2].

Theorem 2.1. David [4] If the distortion function $K(z)$ satisfies

$$
|\{z \in \mathbf{C}: K(z)>t\}|=\mathcal{O}\left(e^{-p t}\right)
$$

for a constant $p>0$, the Beltrami equation $f_{\bar{z}}=\mu(z) f_{z}$ has a principal $W_{\text {loc }}^{1,1}$-solution. Moreover, the principal homeomorphism maps sets of Lebesgue measure zero to sets of measure zero.

The homeomorphic solution in Theorem 2.1 is usually called the David homeomorphism. The more general solutions of the degenerate Beltrami equation was developed by Iwaniec and Martin [3, 6].

Theorem 2.2. [3, Theorem 20.5.2] Suppose $\mathcal{A}(x)$ is a strictly increasing smooth function with $\mathcal{A}(1)=1$, which satisfies that $\int_{1}^{+\infty} \frac{\mathcal{A}(x)}{x^{2}} d x=+\infty$ and $\lim _{x \rightarrow+\infty} x \mathcal{A}^{\prime}(x)$ $=+\infty$. If the distortion function $K(z)$ satisfies

$$
e^{\mathcal{A}(K(z))} \in L_{\mathrm{loc}}^{1}(\mathbf{C}),
$$

the Beltrami equation $f_{\bar{z}}=\mu(z) f_{z}$ admits a unique principal solution $f \in W_{\text {loc }}^{1, P}(\mathbf{C})$ with $P(t)=\frac{t^{2}}{\mathcal{A}^{-1}\left(\log t^{2}\right)}$. Moreover, any solution $h \in W_{\text {loc }}^{1, P}(\mathbf{C})$ to this Beltrami equation admits a factorization

$$
h=\phi \circ f,
$$

where $\phi$ is a holomorphic function.

Here $W_{l o c}^{1, P}(\mathbf{C})$ is the Sobolev-Orlicz space with the regularity function $P(x)$. For its definition, we first introduce the Orlicz space.

A Young function $A$ is a strictly increasing convex function. The Orlicz space $L^{A}(\Omega)$ is defined as

$$
L^{A}(\Omega)=\left\{u \in L^{1}(\Omega): \exists \lambda>0 \text { s.t. } \int_{\Omega} A\left(\frac{|u|}{\lambda}\right)<+\infty\right\},
$$

which is a Banach space equipped with the Luxemburg norm, $\|u\|_{L^{A}(\Omega)}=\inf \{k>$ $\left.0: \int_{\Omega} A\left(\frac{|u|}{k}\right) \leq 1\right\}$. If the Young function $A(t)$ is doubling (i.e. $A(2 t)<c A(t)$ ), the Orlicz space coincides with the set

$$
L^{A}(\Omega)=\left\{u \in L^{1}(\Omega): \int_{\Omega} A(|u|)<+\infty\right\} .
$$

The Young conjugate is defined as $\widetilde{A}(s)=\sup \{s t-A(t): t \geq 0\}$. When the Young function $A(t)$ and its Young conjugate $\widetilde{A}$ are both doubling, the Orlicz space $L^{A}(\Omega)$ is reflexive. In particular, every bounded sequence has a weakly convergent subsequence.

The Orlicz-Sobolev space $W_{\text {loc }}^{1, A}(\Omega)$ is the set of functions $u(z) \in W_{\text {loc }}^{1,1}(\Omega)$ for which $|D u(z)| \in L_{\text {loc }}^{A}(\Omega)$, where $|D u(z)|$ is the operator norm of the first generalized derivatives $D u(z)$. 
Let $\psi(t)$ be the inverse function of $t=\mathcal{A}(x)$ in Theorem 2.2 and let $\varphi(t)=$ $\psi(\log t)$. The function $\varphi(t)$ is strictly increasing on $[e,+\infty)$ with $\varphi(e)=1$. We have

$$
\lim _{t \rightarrow+\infty} \frac{t \varphi^{\prime}(t)}{\varphi(t)}=\lim _{t \rightarrow+\infty} \frac{\psi^{\prime}(t)}{\psi(t)}=\lim _{x \rightarrow+\infty} \frac{1}{x \mathcal{A}^{\prime}(x)}=0
$$

and

Now define two functions

$$
\int_{e}^{+\infty} \frac{1}{t \varphi(t)} d t=\int_{1}^{+\infty} \frac{1}{\psi(t)} d t=+\infty
$$

$$
P(t)=\frac{t^{2}}{\mathcal{A}^{-1}\left(\log t^{2}\right)}=\frac{t^{2}}{\varphi\left(t^{2}\right)}, \quad \Phi(t)=\int_{e}^{t^{2}} \frac{1}{\varphi(s)} d s .
$$

We have $W_{\text {loc }}^{1, P}(\mathbf{C})=W_{\text {loc }}^{1, \Phi}(\mathbf{C})$. In fact, $P(t)$ and $\Phi(t)$ are comparable near $\infty$. Here we give the details for completeness. For $\alpha>0$, we have

$$
\left(\frac{t^{\alpha}}{\varphi(t)}\right)^{\prime}=\frac{\alpha t^{\alpha-1} \varphi(t)-t^{\alpha} \varphi^{\prime}(t)}{\varphi^{2}(t)}=\frac{t^{\alpha-1}}{\varphi(t)}\left(\alpha-\frac{t \varphi^{\prime}(t)}{\varphi(t)}\right)>0 .
$$

So $\frac{\varphi(t)}{t^{\alpha}}$ is strictly decreasing, which implies $\lim _{t \rightarrow \infty} \frac{\varphi(t)}{t^{\alpha}}=0$. Consider the integral

$$
\int_{e}^{t} \frac{1}{\varphi(s)} d s=\frac{t}{\varphi(t)}-e+\int_{e}^{t} \frac{s \varphi^{\prime}(s)}{\varphi^{2}(s)} d s
$$

Since $\lim _{t \rightarrow+\infty} \frac{t \varphi^{\prime}(t)}{\varphi(t)}=0$, we can assume $\frac{t \varphi^{\prime}(t)}{\varphi(t)}<\varepsilon$ for $t>t(\varepsilon)$. Then

$$
\int_{e}^{t} \frac{s \varphi^{\prime}(s)}{\varphi^{2}(s)} d s=\int_{e}^{t(\varepsilon)} \frac{s \varphi^{\prime}(s)}{\varphi^{2}(s)} d s+\int_{t(\varepsilon)}^{t} \frac{s \varphi^{\prime}(s)}{\varphi^{2}(s)} d s \leq M_{\varepsilon}+\varepsilon \int_{e}^{t} \frac{1}{\varphi(s)} d s .
$$

Thus we have

$$
\frac{t}{\varphi(t)}-e \leq \int_{e}^{t} \frac{1}{\varphi(s)} d s \leq \frac{1}{1-\varepsilon}\left(\frac{t}{\varphi(t)}-e+M_{\varepsilon}\right)
$$

which implies $\lim _{t \rightarrow+\infty} \frac{\int_{e}^{t} \frac{1}{\varphi(s)} d s}{\frac{t}{\varphi(t)}}=1$ since $\lim _{t \rightarrow \infty} \frac{t}{\varphi(t)}=\infty$. Therefore we have $\lim _{t \rightarrow+\infty} \frac{P(t)}{\Phi(t)}=1$.

The function $\Phi(t)$ satisfies the condition $\int_{\sqrt{e}}^{+\infty} \frac{\Phi(t)}{t^{3}} d t=+\infty$, which is the corollary of the following equality

$$
\int_{\sqrt{e}}^{+\infty} \frac{P(t)}{t^{3}} d t=\int_{\sqrt{e}}^{+\infty} \frac{1}{t \varphi\left(t^{2}\right)} d t=\frac{1}{2} \int_{e}^{+\infty} \frac{1}{t \varphi(t)} d t=+\infty .
$$

Furthermore the functions $\Phi\left(t^{\frac{5}{8}}\right)$ and $\Phi(t)$ are convex near $\infty$. Indeed, for $\alpha>0$, we have

$$
\frac{d}{d t} \Phi\left(t^{\frac{1}{2}+\alpha}\right)=\frac{d}{d t}\left(\int_{e}^{t^{1+2 \alpha}} \frac{1}{\varphi(s)} d s\right)=(1+2 \alpha) \frac{t^{2 \alpha}}{\varphi\left(t^{1+2 \alpha}\right)} .
$$

Since $\frac{t^{\alpha}}{\varphi(t)}$ is strictly increasing, the function $\Phi\left(t^{\frac{1}{2}+\alpha}\right)$ is convex near $\infty$.

The function $\Phi(t)$ and the Young conjugate $\widetilde{\Phi}(s)$ are doubling. So the Orlicz space $L^{\Phi}(\Omega)$ is reflexive and coincides with the set $\left\{u(z): \int_{\Omega} \Phi(|u|)<+\infty\right\}$. The following is the details. The derivatives of $\Phi$ satisfy that

$$
\Phi^{\prime}(t)=\frac{2 t}{\varphi\left(t^{2}\right)}, \quad \Phi^{\prime \prime}(t)=2 \frac{\varphi\left(t^{2}\right)-2 t^{2} \varphi^{\prime}\left(t^{2}\right)}{\varphi^{2}\left(t^{2}\right)} .
$$


Then

$$
\lim _{t \rightarrow+\infty} \frac{\Phi^{\prime}(t) t}{\Phi(t)}=\lim _{t \rightarrow+\infty} \frac{\Phi^{\prime}(t)-t \Phi^{\prime \prime}(t)}{\Phi^{\prime}(t)}=\lim _{t \rightarrow+\infty} 2 \frac{t^{2} \varphi^{\prime}\left(t^{2}\right)}{\varphi\left(t^{2}\right)}=0 .
$$

Since

$$
\frac{\Phi(2 t)-\Phi(t)}{\Phi(2 t)}=\frac{\Phi^{\prime}(\xi) t}{\Phi(2 t)}<\frac{\Phi^{\prime}(2 t) t}{\Phi(2 t)}=\frac{1}{2} \frac{\Phi^{\prime}(2 t) 2 t}{\Phi(2 t)}
$$

we have $\lim _{t \rightarrow+\infty} \frac{\Phi(2 t)}{\Phi(t)}=1$.

The function $\Phi(t)$ and its Young conjugate $\widetilde{\Phi}(s)$ satisfy $\widetilde{\Phi}^{\prime}\left(\Phi^{\prime}(t)\right)=t$ by definition. In fact, if $\widetilde{\Phi}(s)=s t-\Phi(t)$, we have $0=s-\Phi^{\prime}(t)$, which yields

$$
\widetilde{\Phi}\left(\Phi^{\prime}(t)\right)=\Phi^{\prime}(t) t-\Phi(t) .
$$

By taking the derivative, we obtain the equality above. Since

$$
\lim _{s \rightarrow+\infty} \frac{\widetilde{\Phi}^{\prime}(s) s}{\widetilde{\Phi}(s)}=\lim _{t \rightarrow+\infty} \frac{\widetilde{\Phi}^{\prime}\left(\Phi^{\prime}(t)\right) \Phi^{\prime}(t)}{\widetilde{\Phi}\left(\Phi^{\prime}(t)\right)}=\lim _{t \rightarrow+\infty} \frac{t \Phi^{\prime}(t)}{\Phi^{\prime}(t) t-\Phi(t)}=0,
$$

we have $\lim _{s \rightarrow+\infty} \frac{\widetilde{\Phi}(2 s)}{\widetilde{\Phi}(s)}=1$.

The Orlicz-Sobolev space in Theorem 2.2 satisfies $W_{\text {loc }}^{1, P}(\mathbf{C}) \subset W_{\text {loc }}^{1, p}(\mathbf{C})$ for $1 \leq$ $p<2$. Indeed, the function $\frac{t^{\alpha}}{\varphi(t)}$ is strictly increasing for $\alpha>0$, then we have $\frac{x^{2}}{\varphi\left(x^{2}\right)}>c x^{p}$.

The following theorem shows that the Jacobian of the function in $W_{l o c}^{1, P}(\mathbf{C})$ is locally integrable. Recalled that $W_{\mathrm{loc}}^{1, P}(\mathbf{C})=W_{\mathrm{loc}}^{1, \Phi}(\mathbf{C})$ and the function $\Phi(t)$ satisfies that $\Phi\left(t^{\frac{5}{8}}\right)$ is convex near $\infty$ and $\int_{\sqrt{e}}^{+\infty} \frac{\Phi(t)}{t^{3}} d t=+\infty$.

Theorem 2.3. [6, Theorem 7.2.1] Let $f=u+i v \in W_{\text {loc }}^{1, P}(\mathbf{C})$ be an orientationpreserving mapping. Then the Jacobian $J(z, f)$ is locally integrable and

$$
\int_{\mathbf{C}} \eta(z) J(z, f)=-\int_{\mathbf{C}} u(z) d \eta \wedge d v
$$

for every test function $\eta \in C_{0}^{\infty}(\mathbf{C})$.

By Theorem 2.3, every solution in $W_{\text {loc }}^{1, P}(\mathbf{C})$ in Theorem 2.2 has locally integrable Jacobian. A function $f(z)$ on a domain $\Omega \subset \mathbf{C}$ is said to be of finite distortion if the following three conditions are satisfied:

(1) $f \in W_{\text {loc }}^{1,1}(\Omega)$

(2) $J(z, f) \in L_{\text {loc }}^{1}(\Omega)$, and

(3) there is a measurable function $K(z) \geq 1$, finite almost everywhere, such that

$$
|D f(z)|^{2} \leq K(z) J(z, f) \quad \text { almost everywhere in } \Omega .
$$

Let $f(z) \in W_{\text {loc }}^{1,1}(\mathbf{C})$ be a solution to the Beltrami equation $f_{\bar{z}}=\mu(z) f_{z}$. If $|\mu(z)|<1$ holds for almost every $z \in \mathbf{C}$, the condition (3) is satisfied automatically. Then $f(z) \in W_{\text {loc }}^{1,1}(\mathbf{C})$ is a solution of finite distortion if and only if it has locally integrable Jacobian. Note that a homeomorphism $f(z) \in W_{\text {loc }}^{1,1}(\mathbf{C})$ has locally integrable Jacobian, in particular, it is a mapping of finite distortion. For more properties of the mapping of finite distortion one can see [5]. 


\section{Solutions of the degenerate Beltrami equation}

First we give a reformulation of Theorem 2.2 by the following theorem. It is exactly Theorem 2.1 of David [4] if we take the function $v(t)=1$ in Theorem 3.1.

Theorem 3.1. Let $v(t)>0$ be a concave function such that $\int_{1}^{+\infty} \frac{1}{t v(t)} d t=+\infty$. If the distortion function $K(z)$ satisfies

$$
|\{z \in \mathbf{C}: K(z)>t v(t)\}|=\mathcal{O}\left(e^{-p t}\right)
$$

for a constant $p>0$, the Beltrami equation $f_{\bar{z}}=\mu(z) f_{z}$ admits a unique principal solution of finite distortion. Moreover, any solution of finite distortion to this Beltrami equation admits a factorization

$$
h=\phi \circ f,
$$

where $\phi$ is a holomorphic function.

The solution of finite distortion to this Beltrami equation lies in the SobolevOrlicz space $W_{\operatorname{loc}}^{1, P}(\mathbf{C})$, where $P(t)=\frac{t^{2}}{v(\log t) \log t}$. The functions $\log t, \log t \log \log t, \cdots$ satisfy the condition in Theorem 3.1 .

Proof. Since $v(t)>0$ is a concave function, then $v(t)$ is increasing. If $\lim _{t \rightarrow+\infty} v(t)$ $<+\infty$, the conclusion holds by Theorem 2.1 of David. So we assume that $\lim _{t \rightarrow+\infty} v(t)$ $=+\infty$ in the following. Further assume that $v(1)=1$.

By assumption, the distortion function $K(z)$ satisfies

$$
|\{z \in \mathbf{C}: K(z)>t v(t)\}|<c e^{-p t},
$$

where $c>0$ and $0<p<1$. Let $t=\frac{1}{q} \log x$ with $0<q<p$. It becomes

$$
\left|\left\{z \in \mathbf{C}: K(z)>\frac{1}{q} \log x v\left(\frac{1}{q} \log x\right)\right\}\right|<c\left(\frac{1}{x}\right)^{\frac{p}{q}} .
$$

Let $\psi(t)=\frac{t}{q} v\left(\frac{t}{q}\right)$. It satisfies $\lim _{t \rightarrow+\infty} \frac{t}{\psi(t)}=0$ and $\int_{1}^{+\infty} \frac{1}{\psi(t)} d t=+\infty$. Let $\mathcal{A}(x)$ be the inverse function of $x=\psi(t)$. Then the distortion function $K(z)$ satisfies

$$
\left|\left\{z \in \mathbf{C}: e^{\mathcal{A}(K(z))}>x\right\}\right|<c\left(\frac{1}{x}\right)^{\frac{p}{q}} .
$$

Applying the Fubini's theorem on a bounded domain $\Omega \subset \mathbf{C}$, we have

$$
\begin{aligned}
\int_{\Omega} e^{\mathcal{A}(K(z))} d \sigma & =\int_{\Omega} d \sigma \int_{1}^{e^{\mathcal{A}(K(z))}} d x=\int_{1}^{+\infty} d t \int_{\left\{z \in \Omega: e^{\mathcal{A}(K(z))}>x\right\}} d \sigma \\
& \leq \int_{1}^{+\infty} c\left(\frac{1}{x}\right)^{\frac{p}{q}} d x<+\infty
\end{aligned}
$$

So the distortion function $K(z)$ satisfies $e^{\mathcal{A}(K(z))} \in L_{\text {loc }}^{1}(\mathbf{C})$.

The function $\mathcal{A}(x)$ satisfies that

$$
\lim _{x \rightarrow+\infty} \frac{1}{x \mathcal{A}^{\prime}(x)}=\lim _{t \rightarrow+\infty} \frac{\psi^{\prime}(t)}{\psi(t)}=\lim _{t \rightarrow+\infty}\left(\frac{1}{t}+\frac{1}{q} \frac{v^{\prime}\left(\frac{t}{q}\right)}{v\left(\frac{t}{q}\right)}\right)=0,
$$

where the last equality is because $v^{\prime}(t)$ is decreasing and $\lim _{t \rightarrow+\infty} v(t)=+\infty$. Next,

$$
\int_{1}^{+\infty} \frac{\mathcal{A}(x)}{x^{2}} d x=-\left.\frac{\mathcal{A}(x)}{x}\right|_{1} ^{+\infty}+\int_{1}^{+\infty} \frac{\mathcal{A}^{\prime}(x)}{x} d x=-\left.\frac{\mathcal{A}(x)}{x}\right|_{1} ^{+\infty}+\int_{1}^{+\infty} \frac{1}{\psi(t)} d t .
$$


Since $\lim _{x \rightarrow+\infty} \frac{\mathcal{A}(x)}{x}=\lim _{t \rightarrow+\infty} \frac{t}{\psi(t)}=0$ and $\int_{1}^{+\infty} \frac{1}{\psi(t)} d t=+\infty$, we have $\int_{1}^{+\infty} \frac{\mathcal{A}(x)}{x^{2}} d x=$ $+\infty$.

Therefore the function $\mathcal{A}(x)$ and $K(z)$ satisfy the conditions of Theorem 2.2. Then the Beltrami equation $f_{\bar{z}}=\mu f_{z}$ has a unique principal solution $f \in W_{\text {loc }}^{1, P}(\mathbf{C})$ with

$$
P(t)=\frac{t^{2}}{\psi\left(\log t^{2}\right)}=\frac{t^{2}}{\frac{1}{q} \log t^{2} v\left(\frac{1}{q} \log t^{2}\right)} .
$$

By Theorem 2.3, it has locally integrable Jacobian, then it is a mapping of finite distortion. The regularity function $P(t)$ can be simplified by an equivalent function $\frac{t^{2}}{v(\log t) \log t}$. In fact, the functions $v(\alpha t)$ and $v(t)$ are comparable. That is, for a constant $\alpha>0$, there is a constant $c^{\prime}>1$ such that $\frac{1}{c^{\prime}} v(t) \leq v(\alpha t) \leq c^{\prime} v(t)$. The following is the detail. If $\alpha>1$, we have

$$
\frac{v(\alpha t)-v(\alpha)}{v(t)-v(1)}=\alpha \frac{v^{\prime}(\alpha \xi)}{v^{\prime}(\xi)} \leq \alpha .
$$

Thus $v(t) \leq v(\alpha t) \leq c^{\prime} v(t)$. If $\alpha<1$, we have $v(\alpha t) \leq v\left(\frac{1}{\alpha}(\alpha t)\right) \leq c^{\prime} v(\alpha t)$. So we always have $\frac{1}{c} v(t) \leq v(\alpha t) \leq c^{\prime} v(t)$.

Conversely, let $h(z)$ be a solution of finite distortion to this Beltrami equation. The Jacobian $J(z, h)$ is locally integrable by definition. Let $\varphi(t)=\psi(\log t)$. The function $\frac{t}{\varphi(t)}$ is increasing as shown before. For each $K \geq 1$, by considering the two cases, either $t^{2} \leq \varphi^{-1}(K)$ or $t^{2}>\varphi^{-1}(K)$, we always have

$$
\frac{t^{2}}{\varphi\left(t^{2}\right)} \leq \frac{\varphi^{-1}(K)}{K}+\frac{t^{2}}{K}
$$

which yields $\frac{t^{2}}{\varphi\left(t^{2}\right)} \leq \varphi^{-1}(K)+\frac{t^{2}}{K}$. Then

$$
\frac{|D h|^{2}}{\psi\left(\log |D h|^{2}\right)} \leq e^{\mathcal{A}(K(z))}+\frac{|D h|^{2}}{K(z, h)}=e^{\mathcal{A}(K(z))}+J(z, h) .
$$

Thus the solution $h$ lies in $W_{\text {loc }}^{1, P}(\mathbf{C})$. By Theorem 2.2, there is a holomorphic function $\phi$ such that $h=\phi \circ f$.

The following corollary is used in the proof of Theorem 1.1.

Corollary 3.2. Let $v(t)>0$ be a concave function such that $\int_{1}^{+\infty} \frac{1}{t v(t)} d t=+\infty$. If the area

$$
|\{z \in \mathbf{C}: K(z)>k v(k)\}|=\mathcal{O}\left(e^{-p k}\right), \quad k=1,2, \cdots,
$$

for a constant $p>0$, the Beltrami equation $f_{\bar{z}}=\mu(z) f_{z}$ admits a unique principal solution of finite distortion.

Proof. Let $A(t)$ be the area of the set $\{z \in \mathbf{C}: K(z)>t v(t)\}$. In particular, $A(t) \leq A(k)$ for every $k \leq t<k+1$. In particular, the area function $A(t)$ satisfies

$$
A(t) \leq A_{k}<c e^{-p k}<c e^{-p(t-1)} .
$$

So the corollary holds by Theorem 3.1.

Next we prove that the solution of finite distortion to the Beltrami equation in Theorem 3.1 satisfies the Lusin's condition $\mathcal{N}$.

Theorem 3.3. Let $h(z)$ be a solution of finite distortion to the Beltrami equation $f_{\bar{z}}=\mu(z) f_{z}$ in Theorem 3.1. Then $h(z)$ maps sets of Lebesgue measure zero to sets of measure zero. 
Proof. By Theorem 3.1, the solution $h(z)$ of finite distortion to the Beltrami equation $f_{\bar{z}}=\mu(z) f_{z}$ admits a factorization

$$
h=\phi \circ f,
$$

where $\phi$ is an analytic function. The analytic function maps sets of Lebesgue measure zero to sets of measure zero. So we only need prove the theorem for the principal solution $f(z)$, which is a homeomorphic solution.

Let $v(t)>0$ be the concave function with $v(1)=1$ and $\int_{1}^{+\infty} \frac{1}{t v(t)} d t=+\infty$. The distortion function $K(z)$ satisfies

$$
|\{z \in \mathbf{C}: K(z)>t v(t)\}|=\mathcal{O}\left(e^{-p t}\right)
$$

for a constant $p>0$ by the assumption. Let $\mathcal{A}(x)$ be the inverse function of $x=t v(t)$.

Let $\rho_{\mathbf{D}}$ be the hyperbolic metric on the unit disk $\mathbf{D}$. The distortion function of $\mu$ satisfies $K_{\mu}(z)=e^{\rho_{\mathbf{D}}(0, \mu(z))}$. For each $z \in \mathbf{D}$, let $d(z)=\mathcal{A}\left(e^{\rho_{\mathbf{D}}(0, \mu(z))}\right)$, which implies that $e^{\rho_{\mathbf{D}}(0, \mu(z))}=\left.t v(t)\right|_{t=d(z)}$.

Now we define another Beltrami coefficient $\nu(z)$. If $d(z) \leq 1$, we take $\nu(z)=0$. Otherwise, we take $\nu(z)$ to be a point on the geodesic line between 0 and $\mu(z)$ such that

$$
e^{\rho_{\mathbf{D}}(0, \nu(z))}=d(z)
$$

As a result, we have

$$
e^{\rho_{\mathbf{D}}(\nu(z), \mu(z))}=v(d(z))
$$

which follows the equality $e^{\rho_{\mathbf{D}}(0, \nu(z))} e^{\rho_{\mathbf{D}}(\nu(z), \mu(z))}=e^{\rho_{\mathbf{D}}(0, \mu(z))}=\left.t v(t)\right|_{t=d(z)}$. The condition

is equivalent to

$$
\left|\left\{z \in \mathbf{C}: K_{\mu}(z)>t v(t)\right\}\right|=\mathcal{O}\left(e^{-p t}\right),
$$

Then we have

$$
|\{z \in \mathbf{C}: d(z)>t\}|=\mathcal{O}\left(e^{-p t}\right)
$$

$$
\left|\left\{z \in \mathbf{C}: e^{\rho_{\mathbf{D}}(0, \nu(z))}>t\right\}\right|=\mathcal{O}\left(e^{-p t}\right),
$$

and

$$
\left|\left\{z \in \mathbf{C}: e^{\rho_{\mathbf{D}}(\nu(z), \mu(z))}>v(t)\right\}\right|=\mathcal{O}\left(e^{-p t}\right) .
$$

Solving the Beltrami equation $g_{\bar{z}}=\nu g_{z}$, we will obtain a David homeomorphism $g(z)$ by Theorem 2.1. Define another Beltrami coefficient

$$
\kappa(w)=\frac{\mu(z)-\nu(z)}{1-\overline{\nu(z)} \mu(z)}\left(\frac{\partial_{z} g}{\left|\partial_{z} g\right|}\right)^{2}, \quad w=g(z), z \in \mathbf{C},
$$

which satisfies that

$$
\frac{1+|\kappa|}{1-|\kappa|}=\frac{1+\left|\frac{\mu-\nu}{1-\bar{\nu} \mu}\right|}{1-\left|\frac{\mu-\nu}{1-\bar{\nu} \mu}\right|}=e^{\rho_{\mathbf{D}}(\nu(z), \mu(z))} .
$$

In particular, we have $\left|\left\{w \in \mathbf{C}: K_{\kappa}(w)>v(t)\right\}\right|=\mathcal{O}\left(e^{-p t}\right)$. Since $v(t)$ is concave, we have $v(t) \leq c t$. Then the distortion function $K_{\kappa}$ satisfies that

$$
\left|\left\{w \in \mathbf{C}: K_{\kappa}(w)>c t\right\}\right|=\mathcal{O}\left(e^{-p t}\right) .
$$

Also by Theorem 2.1 we can solve the Beltrami equation $F_{\bar{z}}=\kappa F_{z}$ to obtain a David homeomorphism $F(z)$. Since $\mu_{F}=\mu_{f \circ g^{-1}}$, by the uniqueness part in Theorem 2.2 we have

$$
F=\phi \circ f \circ g^{-1}
$$

where $\phi$ is a conformal mapping. 
As a result, the mapping $f=\phi^{-1} \circ F \circ g$ decomposes to a conformal mapping and two David homeomorphisms, which all maps sets of Lebesgue measure zero to sets of measure zero. Then $f(z)$ also maps sets of Lebesgue measure zero to sets of measure zero.

By Theorem 1.3 in [5], a homeomorphism of finite distortion $f \in W_{\text {loc }}^{1,1}(\Omega)$ with $K(z) \in L^{1}(\Omega)$ satisfies that $f^{-1} \in W_{\text {loc }}^{1,2}(f(\Omega))$. Then $f^{-1}$ also satisfies the Lusin's condition $\mathcal{N}$. As a result, both of $f$ and $f^{-1}$ are absolutely continuous and map the Lebesgue measurable set to the Lebesgue measurable set. Furthermore,

$$
|f(E)|=\int_{E} J(z, f)
$$

for any measurable set $E$.

\section{The quadratic polynomial with a Siegel disk}

Let $P_{\theta}(z)=e^{2 \pi i \theta} z+z^{2}$ be a quadratic polynomial, where $0<\theta<1$ is an irrational number with the continued fraction expansion $\theta=\left[0 ; a_{1}, a_{2}, \cdots, a_{k}, \cdots\right]$. Consider the Douady-Ghys Blaschke model

$$
f_{\theta}(z)=e^{2 \pi i t} z^{2} \frac{z-3}{1-3 z}
$$

Here $0<t=t(\theta)<1$ is the unique parameter for which the critical circle map $\left.f_{\theta}\right|_{\mathbf{S}^{1}}: \mathbf{S}^{1} \rightarrow \mathbf{S}^{1}$ has rotation number $\theta$. The function $f_{\theta}$ has a double critical point at $z=1$ and it is a critical circle map, i.e., a real-analytic homeomorphism of $\mathbf{S}^{1}$ with a critical point. It was proved by Yoccoz [12] that a critical circle map with an irrational rotation number can be topologically conjugated with the rigid rotation $R_{\theta}(z)=e^{2 \pi i \theta} z$.

Theorem 4.1. (Yoccoz) Let $f: \mathbf{S}^{1} \rightarrow \mathbf{S}^{1}$ be a critical circle map with irrational rotation number $\theta$. Then there exists a homeomorphism $h: \mathbf{S}^{1} \rightarrow \mathbf{S}^{1}$ such that $f=h^{-1} \circ R_{\theta} \circ h$. This $h$ is uniquely determined once normalized by $h(1)=1$.

Furthermore by the construction of Yoccoz (see the appendix of [10]), $h$ can be extended to a homeomorphism $H: \mathbf{D} \rightarrow \mathbf{D}$ with the controlled dilatation. The construction bases on two cell decompositions of unit disk $\mathbf{D}$, denoted by $\Gamma$ and $\Gamma^{\prime}$. The cells in the decompositions are labeled by some integers, called their level. The closer the cell is chosen to the boundary of unit disk $\mathbf{D}$, the higher its level and the smaller its Euclidean diameter will be. The homeomorphism $H(z)$ maps each cell $\gamma$ of level $n$ in $\Gamma$ to a unique cell $\gamma^{\prime}$ of level $n$ in $\Gamma^{\prime}$. The area of cells in $\Gamma$ and the dilatation of $H(z)$ are controlled by the following theorem of Yoccoz. More details can be found in the Theorem 6.5 in the appendix of [10].

Theorem 4.2. (Yoccoz) The area of the union of all the $m$-cells for all $m>k$ satisfies that

$$
\operatorname{area}\left(\bigcup_{m=k}^{\infty}\{\gamma: \gamma \text { is an } m \text {-cell of } \Gamma\}\right)=\mathcal{O}\left(\sigma^{k}\right)
$$

for a universal constant $0<\sigma<1$. For any $k$-cell $\gamma$ of $\Gamma$, the homeomorphism $H: \gamma \rightarrow$ $\gamma^{\prime}$ is a quasiconformal mapping with the dilatation $K\left(\left.H\right|_{\gamma}\right)=\mathcal{O}\left(1+\left(\log a_{k+1}\right)^{2}\right)$.

We assume $H(0)=0$ and define

$$
F(z)= \begin{cases}f_{\theta} & \text { if }|z| \geq 1 \\ H^{-1} \circ R_{\theta} \circ H & \text { if }|z|<1\end{cases}
$$


Then $F$ is a degree 2 topological branched covering of the sphere. It is holomorphic outside of $\mathbf{D}$ and is topologically conjugate to $R_{\theta}$ on $\mathbf{D}$. The "Julia set" $J(F)$ is defined to be the closure of the set

$$
\left\{z \in \mathbf{C}: F^{\circ n}(z) \in \mathbf{S}^{1} \text { for some } n \geq 1\right\}
$$

which serves as a model for the Julia set $J\left(P_{\theta}\right)$. The following theorem on the property of the Julia set $J(F)$ can be found in $[9,11]$.

Theorem 4.3. (Petersen, Lyubich) For every irrational $0<\theta<1$ the Julia set $J(F)$ is locally connected and has Lebesgue measure zero.

If the coefficient $a_{k}$ in the continued fraction expansion of $\theta$ satisfies that $\log ^{2} a_{k}=$ $\mathcal{O}(k)$, Petersen and Zakeri [10] proved that the Julia set of the quadratic polynomial $P_{\theta}(z)=e^{2 \pi i \theta} z+z^{2}$ is locally connected and has Lebesgue measure zero. The crucial part in their proof is the following area estimation.

Theorem 4.4. [10, Theorem B] There exist a universal constant $0<\beta<1$ and a constant $C>0$ (depending on $\theta$ ) such that

$$
\operatorname{area}(E)+\sum_{k} \operatorname{area}\left(F_{\theta}^{-k}(E)\right) \leq C(\operatorname{area}(E))^{\beta} .
$$

for every measurable set $E \subset \mathbf{D}$.

Now we can prove the Theorem 1.1 by a classical argument as in [10].

Proof of the Theorem 1.1. First pull back $\mu_{H}(z)$ by the mapping $F(z)$ and define a measurable function $\mu(z)$ :

$$
\mu(z)= \begin{cases}\mu_{H}(z) & \text { if } z \in \mathbf{D}, \\ \mu_{H}\left(F^{\circ n}(z)\right) \frac{\overline{\partial_{z} F^{\circ n}(z)}}{\partial_{z} F^{\circ n}(z)} & \text { if } z \notin \mathbf{D} \text { and } F^{\circ n}(z) \in \mathbf{D}, \\ 0 & \text { elsewhere. }\end{cases}
$$

Let $\gamma$ be a cell of level $k$ in $\Gamma$. The dilatation of the homeomorphism $H(z)$ on $\gamma$ satisfies that $K\left(\left.H\right|_{\gamma}\right)=\mathcal{O}\left(1+\left(\log a_{k+1}\right)^{2}\right)$ by the Theorem 4.2. By assumption $\log ^{2} a_{k} \leq k v(k)$, where $v(t)>0$ a concave function such that $\int_{1}^{+\infty} \frac{1}{t v(t)} d t=+\infty$. Then there is a constant $C_{1}$ independent of $k$ such that $K\left(\left.H\right|_{\gamma}\right)<C_{1} k v(k)$. Therefore we have

$$
\left\{z \in \mathbf{D}: K_{H}(z)>C_{1} k v(k)\right\} \subset \bigcup_{m=k}^{\infty}\{\gamma: \gamma \text { is an } m \text {-cell of } \Gamma\} .
$$

By the Theorem 4.2, the area satisfies area $\left(\bigcup_{m=k}^{\infty}\{\gamma: \gamma\right.$ is an $m$-cell of $\left.\Gamma\}\right)=\mathcal{O}\left(\sigma^{k}\right)$, which yields

$$
\operatorname{area}\left(\left\{z \in \mathbf{D}: K_{H}(z)>C_{1} k v(k)\right\}\right)=\mathcal{O}\left(\sigma^{k}\right) .
$$

By the Theorem 4.4, the dilatation function $K(z)$ of $\mu(z)$ satisfies that

$$
\operatorname{area}\left(\left\{z \in \mathbf{C}: K(z)>C_{1} k v(k)\right\}\right) \leq C\left(\operatorname{area}\left(\left\{z \in \mathbf{D}: K_{H}(z)>C_{1} k v(k)\right\}\right)\right)^{\beta},
$$

which implies that for $p=\beta \log \frac{1}{\sigma}$, we have

$$
\operatorname{area}\left(\left\{z \in \mathbf{C}: K(z)>C_{1} k v(k)\right\}\right)=\mathcal{O}\left(e^{-p k}\right) .
$$

The function $\omega(t)=C_{1} v(t)$ is concave with $\int_{1}^{+\infty} \frac{1}{t \omega(t)} d t=+\infty$. By the Corollary 3.2, the Beltrami equation $f_{\bar{z}}=\mu(z) f_{z}$ has a homeomorphic solution $\mathcal{H} \in W_{\text {loc }}^{1,1}(\mathbf{C})$. Now we normalize $\mathcal{H}$ by $\mathcal{H}(0)=0, \mathcal{H}(1)=-e^{2 \pi i \theta} / 2$ and define

$$
P:=\mathcal{H} \circ F \circ \mathcal{H}^{-1} \text {. }
$$


If $z \notin \mathbf{D}$ and $F^{\circ n}(z) \in \mathbf{D}$, the function $F=f_{\theta}$ is analytic. Then

$$
\mu_{\mathcal{H} \circ F}(z)=\mu_{\mathcal{H}}(F(z)) \cdot \frac{\partial_{z} F^{-1}(F(z))}{\overline{\partial_{z} F^{-1}(F(z))}}=\mu_{\mathcal{H}}(F(z)) \cdot \frac{\overline{\partial_{z} F(z)}}{\partial_{z} F(z)}=\mu_{\mathcal{H}}(z) .
$$

If $z \in \mathbf{D}$, the function $F=H^{-1} \circ R_{\theta} \circ H$,

$$
\mathcal{H} \circ F=\mathcal{H} \circ H^{-1} \circ R_{\theta} \circ H .
$$

Since $\left.\mathcal{H} \circ H^{-1}\right|_{\mathbf{D}}$ is conformal, we have $\mu_{\mathcal{H} \circ F}=\mu_{H}$ for $z \in \mathbf{D}$. As a result, $\mu_{\mathcal{H} \circ F}=\mu_{\mathcal{H}}$ for almost every $z \in \mathbf{C}$.

Once we show $\mathcal{H} \circ F \in W_{\text {loc }}^{1,1}(\mathbf{C} \backslash\{1\}), P(z)$ is analytic on $\mathbf{C} \backslash\{1\}$ by the proof of the uniqueness part of Theorem 3.1 since $\mu_{\mathcal{H} \circ F}=\mu_{\mathcal{H}}$. As a result, $P(z)$ is analytic on C since $P(z)$ is continuous on $z=1$. Then by the normalization, $P(z)=e^{2 \pi i \theta} z+z^{2}$. From $P=\mathcal{H} \circ F \circ \mathcal{H}^{-1}$, we obtain that the Julia set $J(P)$ is locally connected and has measure zero by Theorem 4.3.

Now we show that $\mathcal{H} \circ F \in W_{\text {loc }}^{1,1}(\mathbf{C} \backslash\{1\})$. First on $\mathbf{C} \backslash \overline{\mathbf{D}}, \mathcal{H} \circ F=\mathcal{H} \circ f_{\theta}$, so we have $\mathcal{H} \circ F \in W_{\text {loc }}^{1,1}(\mathbf{C} \backslash \overline{\mathbf{D}})$. On the disk $\mathbf{D}$,

$$
\mathcal{H} \circ F=\mathcal{H} \circ H^{-1} \circ R_{\theta} \circ H \in W_{\text {loc }}^{1,1}(\mathbf{D}) .
$$

The mapping $\mathcal{H} \circ H^{-1} \circ R_{\theta}$ is conformal, so we have $\mathcal{H} \circ F \in W_{\text {loc }}^{1,1}(\mathbf{D})$. Finally on a sufficiently small disk $U \subset \mathbf{C} \backslash\{1\}, \mathcal{H} \circ F$ is a homeomorphism which is almost everywhere differentiable, then

$$
\int_{U} J(z, \mathcal{H} \circ F) \leq|\mathcal{H} \circ F(U)|<+\infty \text {. }
$$

As a result,

$$
\begin{aligned}
\int_{U}|D(\mathcal{H} \circ F)| & =\int_{U} \sqrt{K(\mathcal{H} \circ F)} \sqrt{J(\mathcal{H} \circ F)} \\
& \leq\left(\int_{U} J(\mathcal{H} \circ F) \int_{U} K(\mathcal{H} \circ F)\right)^{\frac{1}{2}}<+\infty
\end{aligned}
$$

which implies $\mathcal{H} \circ F \in W^{1,1}(U)$. Then we have $\mathcal{H} \circ F \in W_{\text {loc }}^{1,1}(\mathbf{C} \backslash\{1\})$.

\section{References}

[1] Ahlfors, L. V.: Lectures on quasiconformal mappings. - Van Nostrand, Princeton, 1966; Reprinted by Amer. Math. Soc., 2006.

[2] Astala, K., J. T. Gill, S. Rohde, and E. Saksman: Optimal regularity for planar mappings of finite distortion. - Ann. Inst. H. Poincaré Anal. Non Linéaire 27:1, 2010, 1-19.

[3] Astala, K., T. Iwaniec, and G. Martin: Elliptic partial differential equations and quasiconformal mappings in the plane. - Princeton Math. Ser. 48, Princeton Univ. Press, 2009.

[4] David, G.: Solutions de l'equation de Beltrami avec $\|\mu\|_{\infty}=1$. - Ann. Acad. Sci. Fenn. Ser. A I Math. 13, 1988, 25-70.

[5] Hencl, S., and P. Koskela: Lectures on mappings of finite distortion. - Lecture Notes in Math. 2096, Springer, Cham, 2014.

[6] Iwaniec, T., and G. J. Martin: Geometric function theory and nonlinear analysis. - Oxford Univ. Press, 2001.

[7] Iwaniec, T., and G. J. Martin: The Beltrami equation. - Mem. Amer. Math. Soc. 191, 2008.

[8] Lehto, O., and K. Virtanen: Quasiconformal mappings in the plane. Second edition. Springer-Verlag, 1973. 
[9] Petersen, C. L.: Local connectivity of some Julia sets containing a circle with an irrational rotation. - Acta Math. 177, 1996, 163-224.

[10] Petersen, C. L., and S. Zakeri: On the Julia set of a typical quadratic polynomial with a Siegel disk. - Ann. of Math. (2) 159, 2004, 1-52.

[11] YAmpolsky, M.: Complex bounds for renormalization of critical circle maps. - Ergodic Theory Dynam. Systems 19, 1999, 227-257.

[12] Yoccoz, J.-C.: Il n'y a pas de contre-exemple de Denjoy analytique. - C. R. Acad. Sci. Paris $298,1984,141-144$.

Received 24 November 2016 • Accepted 20 August 2017 